\title{
Traduire
}

Ine autre perspective sur $r$ tadadciction

Revue française de la traduction

$232 \mid 2015$

Intraduisible? Vous voulez rire !

\section{La réinvention calvinienne de l'humour dans Les Fleurs bleues de Raymond Queneau}

Irène Kristeva

\section{(2) OpenEdition}

1 Journals

Édition électronique

URL : https://journals.openedition.org/traduire/696

DOI : $10.4000 /$ traduire.696

ISSN : 2272-9992

Éditeur

Société française des traducteurs

Édition imprimée

Date de publication : 15 juin 2015

Pagination : 56-66

ISSN : 0395-773X

\section{Référence électronique}

Irène Kristeva, «La réinvention calvinienne de l'humour dans Les Fleurs bleues de Raymond Queneau », Traduire [En ligne], 232 | 2015, mis en ligne le 15 juin 2017, consulté le 28 juin 2022. URL : http:// journals.openedition.org/traduire/696 ; DOI : https://doi.org/10.4000/traduire.696 


\section{La réinvention calvinienne de l'humour dans Les Fleurs bleues de Raymond Queneau}

\section{Irène Kristeva}

"Hélas oui, M'sieur, dit Saturin, je travaille de la plume. [...] C'est comme qui dirait une traduction. Oui, je traduis le Discours de la Méthode en argot. " (Raymond Queneau, Parerga notes préparatoires du Chiendent).

En m'appuyant sur cette citation des notes préparatoires du Chiendent, je voudrais, en préambule, poser la question suivante : Pourquoi un écrivain éprouve-t-il le besoin de traduire, c'està-dire de réécrire l'œuvre d'un confrère ? Probablement parce que le contact avec le langage de l'autre aiguise sa sensibilité linguistique, révèle des dimensions inattendues de sa propre langue, la rend plus souple et plus ludique, stimule son expressivité figurative. Ce sont peutêtre quelques-unes des raisons qui ont poussé Italo Calvino à traduire les Fleurs bleues, deux ans après sa parution en France en 1965. D'autre part, le parcours littéraire de l'un des plus grands protagonistes du panorama culturel italien de l'après-guerre, qui couvre de multiples champs allant du néoréalisme au fantastique, témoigne d'une curiosité pour toute tendance innovatrice de la création. La littérature potentielle(1) en fait partie sans aucun doute.

Calvino qui entretient depuis 1964 des relations avec l'OuLiPo, auquel il adhère en 1972, et se lie d'amitié avec certains de ses membres, pour ne citer que Perec et Queneau, relève le défi de traduire une œuvre oulipienne. II faudrait cependant remarquer que la traduction de tout texte de ce type présuppose " une double métamorphose/anamorphose : celle qu'effectue l'auteur et celle que le traducteur est obligé de mettre en œuvre. On y observe des pratiques hypertextuelles, à fonction plus ou moins ludiques (parodie et/ou transposition, pastiche ou réécriture, transformation et imitation)(2). " La traduction des Fleurs bleues est incontestablement une entreprise fort difficile, voire impossible, étant donné qu'il s'agit d'un livre encyclopédique qui met en place la technique de l'écriture sous contrainte en tant que stimulus de l'imagination littéraire.

(1) OuLiPo : OUvroir de LIttérature POtentielle.

(2) Georgiana Lungu Badea, "Traductions transfuges, oulipotentielles ", in Translationes, n 5, 2013, p. 76-77. Lungu Badea précise que si la métamorphose consiste dans la transformation du texte-source, l'anamorphose se manifeste dans sa déformation. 
La version de l'écrivain-traducteur italien demeure encore aujourd'hui, une cinquantaine d'années après sa publication, très respectable, voire admirable de différents points de vue. Calvino fait le jeu de Queneau avec une grande désinvolture. II résout avec sprezzatura (désinvolture) les problèmes du transfert de son "langage privé ", sans l'alourdir par des explicitations inutiles ou des explications superflues. Parmi ces problèmes, la traduction de l'humour occupe une place prépondérante. Essayons de repérer et d'analyser les stratégies traductionnelles calviniennes concernant la réinvention de cet aspect de l'écriture quenaldienne.

\section{Les stratégies de la traduction " réinventive "}

Convaincu que la traduction demande une lecture capable de restaurer les valeurs et les intentions du texte-source, Italo Calvino propose une version qui rend les effets de l'original. Il affronte en pleine conscience les contraintes traductives des Fleurs bleues : le changement constant du registre stylistique dû au dédoublement du personnage du duc d'Auge-Cidrolin, la diversification du discours et les récits parallèles qui en découlent, l'alternance des époques et le grand intervalle temporel qui les sépare (allant de 1264, année de la rencontre du duc d'Auge et de Saint Louis, à 1964, celle de la rencontre du duc et de Cidrolin, mais aussi celle des premiers contacts de l'écrivain italien avec l'OuLiPo), les jeux de mots, les proverbes, les nuances émotionnelles, les citations implicites ou transformées en lieux communs par le texte, les pastiches, les constructions agrammaticales, le parler populaire, etc.(3)

La traduction littéraire observe en général quelques règles herméneutiques : respecter le style et l'expressivité de l'auteur ; suivre le rythme et la musicalité de son œuvre ; reproduire les jeux de mots en cherchant à circonscrire dans la langue-cible les déplacements des signifiants ; éviter les interférences sémantiques et les effets stylistiques qui ne figurent pas dans le texte original ; trouver les expressions qui se rapprochent le plus de celles de l'œuvre à traduire ; forger des néologismes chaque fois que l'original en forge ; restituer le texte-source avec toutes ses omissions, contrariétés, voire éléments inacceptables. Italo Calvino observe grosso modo ces conventions. Mais il n'hésite pas à les transgresser quand il l'estime opportun afin de réinventer d'une façon naturelle et convaincante le monde surréel et divertissant créé par Queneau. Prenons le cas du mot d'esprit, après avoir rappelé que Sigmund Freud entend par ce terme un jugement ludique qui suppose à la fois la condensation et la modification; le caractère métaphorique et démesuré de la condensation est défini comme " un processus dont l'action se prolonge jusqu'à l'arrivée dans la région des perceptions(4) ». En ce qui concerne

(3) Cf. Italo Calvino, "Nota del traduttore ", in Raymond Queneau, I fiori blu, tr. it. di Italo Calvino, Torino, Einaudi tascabili, 1995, p. 263-274.

(4) Sigmund Freud, Le mot d'esprit et ses rapports avec l'inconscient, trad. fr., Paris, Gallimard, 1971, p. 252. 
la modification, elle se présente soit comme une métamorphose, soit comme une anamorphose. La technique du mot d'esprit joue, du reste, sur le double sens du mot modifié, en exploitant la tension entre son sens littéral (manifeste) et son sens métaphorique (caché).

Quant à sa capacité de recréer les séquences de mots d'esprit, Calvino rivalise décidément avec le maître incontesté des calembours(5), Queneau, bien qu'il compense parfois l'impossibilité de refaire le même jeu de mots en italien par un autre. Ainsi, au début du premier chapitre, il traduit " les Sarrasins fauchaient de l'avoine " (Les fleurs bleues, p. 13) par i Saracineschi chiudevano persiane (I fiori blu, p. 3) en substituant au jeu polysémique du verbe français " faucher " (qui signifie " couper le foin ", mais aussi " détruire, voler l'argent ou la femme de quelqu'un ") par le verbe italien chiudere (qui veut dire aussi bien "fermer " [les persiennes] que "capturer "[les femmes persanes]), sans oublier la création du mot Saracineschi (déformation de Saraceni, Sarrasins et saracinesca, rideau métallique). II arrive à transmettre avec brio les jeux linguistiques quenaldiens qui ébranlent l'équilibre du mode commun de penser et de s'exprimer, s'écartent de l'inflexibilité discursive et de la rigueur grammaticale, provoquent l'esprit et stimulent l'imagination. Pourtant, il ne se limite pas à compenser la perte traductionnelle, mais prend le risque de réinventer l'expression. Sa version des Fleurs bleues représente, en fait, une reprise au sens défini par Gérard Genette : "C'est encore le procédé d'autocopie qui distingue les œuvres à répliques des œuvres en relation de ce que j'appelle faute de mieux reprise (remake en anglais)(6). "

À ce texte de prime abord intraduisible, selon son propre jugement(7), Italo Calvino propose une traduction qui semble écrite directement en italien dans un souci d'y faire ressentir les effets de l'original. George Steiner observe déjà dans Après Babel que pour traduire un texte-source d'une manière adéquate, le traducteur, à partir du langage spécifique utilisé dans un contexte particulier, devrait transmettre ces effets dans tout le texte-cible, en différenciant leur place dans l'ensemble des œuvres de l'auteur traduit, en mettant en valeur leur insertion dans le contexte littéraire qui les inspire et les nourrit, voire en accentuant le dépassement du contexte particulier et la recherche des points de référence dans le contexte littéraire universel(8). Sous cet aspect, Calvino ne se heurte pas à des difficultés notables car il connaît à fond aussi bien l'œuvre de Queneau que l'ambiance oulipienne et les enjeux littéraires du temps. Or, la tâche du traducteur se complique davantage quand le texte-source présente une riche intertextualité, provenant d'époques et/ou de cultures différentes, dont il faudrait préserver l'harmonie lexicale, (a)grammaticale et stylistique. C'est effectivement le cas de l'œuvre de Queneau.

(5) À l'origine du calembour on retrouve le mot sanscrit, çlesha, qui signifie " coalescence ". La coalescence présume la condensation qui caractérise le mot d'esprit.

(6) Gérard Genette, L'œuvre de l'art I, Immanence et transcendance, Paris, Seuil, 1994, p. 197.

(7) " È intraducibile ! " - Italo Calvino, " Nota del traduttore ", op. cit., p. 265.

(8) Cf. George Steiner, Après Babel. Une poétique du dire et de la traduction, trad. fr., Albin Michel, 1978, p. 34-37. 
Calvino tient à expliquer son projet dans la " Note du traducteur » incorporée à la deuxième édition de sa version : "La traduzione che qui si ristampa [... è ù un esempio speciale di traduzione "inventiva " (o per meglio dire "reinventiva ") che è l'unico modo d'essere fedeli a un testo di questo tipo(9). "La traduction " réinventive " serait alors l'unique possibilité de rester fidèle au texte aussi bizarre que ludique de Queneau. La traduction " réinventive " devrait faire face au langage particulier, riche et expressif d'un français parlé, parfois difficile à comprendre même par les lecteurs français non avertis. Calvino part donc du présupposé que « la réécriture et l'intertextualité forment une matrice constante. [... ] Le texte s'inscrit dans du texte(10). " Et il affronte avec une conscience surprenante les problèmes posés tant par l'intertextualité que par le "langage très privé " de Queneau et le sens de l'humour dont il est imprégné.

\section{Réinventer I'humour quenaldien}

L'humour, tout comme le rire, est conditionné par la culture, le code sociolinguistique, la mentalité collective, la sensibilité individuelle. La réussite de la traduction de ces deux formes d'esprit dépend à la fois de la pertinence des options proposées pour la langue-culture d'arrivée et de leur capacité à provoquer un effet équivalent à celui qu'on trouve dans la langue-culture de départ. Autrement dit, l'efficacité de la transposition de l'humour est validée par son aptitude à faire rire.

L'humour dans les Fleurs bleues découle de la compréhension discursive, contextuelle et référentielle de plusieurs éléments linguistiques et stylistiques. Relevons-en quelques-uns :

1) Le parler populaire des personnages ("Surtout, vous allez pas faire la course ", p. 61 Però non mettetevi a far corse, p. 50), leur langage familier, voire argotique (" se harnache illico ", p. 22 - s'imbasta del suo basto, p. 12), le néobabélien ("Onivati oder onivatipa ", p. 22 - Cisivà o nosivapyou ?, p. 12), les jeux de mots, les plaisanteries et les proverbes - La maestria de Calvino quant à la traduction de ces dernières réside dans leur réinvention dans la langue-cible, tout en respectant le registre stylistique et en observant le rythme et les rimes internes de chaque dicton, juste avant la césure et à la fin des versets. En voilà quelques exemples : "Animal qu'a parlé, âme damnée " - Bestia articolata, anima dannata ; "Si le coq a ri tôt, l'haricot pue trop. " - Uccello che parla verba volant ; "Quand l'huître a causé, l'huis est très cassé. " - L'ultimo pesce a parlare ha sempre torto ; "À poisson qui cause, petit cochon peu rose. " - A ostrica parlante non si guarda in bocca; " Si bêle le zèbre ut, voilà Belzébuth "Se la zebra dà del tu, s'avvicina Belzebù (Les fleurs bleues, p. 34-35 ; / fiori blu, p. 24). Le

(9) Italo Calvino, "Nota del traduttore ", op. cit., p. 266. (La traduction dans cette réédition [... ] est un exemple particulier de traduction " inventive " (ou mieux, "réinventive ") seul moyen d'être fidèle à un texte de ce genre).

(10) Jean Bollack, Sens contre sens. Comment lit-on?, Paris, éditions La passe du vent, 2000, p. 95. 
rythme de la version de Calvino est légèrement altéré et les rimes ne sont pas toujours observées, mais l'effet désiré par Queneau et la légèreté stylistique sont assurés.

2) Les citations intertextuelles, dissimulées ou transformées en lieux communs - Ainsi, le titre du roman est issu d'une citation d'un vers de "Moesta et errabunda " des Fleurs du mal de Baudelaire, à savoir "Loin ! loin! Ici la boue est faite de nos pleurs ! ", que Queneau modifie légèrement "Loin! loin! ici la boue est faite de nos fleurs. / .. bleues" (p. 15), et Calvino traduit Lontano ! Qui il fango è fatto dei nostri fiori. / ... dei nostri fiori blu (p. 4). En ce qui concerne la traduction littérale du titre, Calvino ne manque pas de justifier sa décision :

La scelta di " blu " anziché " azzurri " m’era parsa piú scattante e queneauiana ma che resta misterioso come significato in rapporto al libro. Mi spiegò [Queneau] il significato francese dell'espressione, che indica ironicamente le persone romantiche, idealiste, nostalgiche d'una purezza perduta, ma non mi diede altri lumi sul valore di questa immagine(11).

\| précise ultérieurement que le mot « blu » lui a semblé plus " vif et plus proche de l'esprit de Queneau(12), "

3) Les abréviations et les constructions agrammaticales - Quant à ces deux catégories, Calvino recourt d'une manière systématique et cohérente à la traduction explicative visant à élucider des références bien connues des Français, mais qui risquent de rester opaques pour les lecteurs italiens. Arrêtons-nous brièvement sur deux néologismes : " ératépiste " et " céhéresses " (p. 53). Le premier substantif est le dérivé du sigle RATP (Régie autonome des transports parisiens) ; le deuxième du sigle CRS (Compagnies républicaines de sécurité). Évidemment, il s'agit de deux realia purement françaises qui n'ont pas d'équivalents dans les autres langues. En traduisant " ératépiste " par dipendente trasporti pubblici et " céhéresses " par guardie (p. 43), Calvino procède à une " clarification ", pour reprendre le terme de Berman, dans le premier cas, et à une simplification dans le second. Cette tendance déformante immanente à la traduction s'applique à expliciter l'implicite. L'humour en souffre, bien entendu, car le référent se perd dans les explications, mais en l'occurrence la perte est inévitable.

4) Les pastiches - Les scènes qui se passent au Bar Biture imitent les romans "chosistes " d'Alain Robbe-Grillet. Ouvrons une parenthèse pour dire que Queneau joue aussi sur la signification explicite du nom du bar qui fait allusion aux sédatifs dont l'usage est très répandu au cours de la première moitié du xxe siècle. Les barbituriques sont des antidépresseurs, ayant

(11) Italo Calvino, "Nota del traduttore ", op. cit., p. 270-271. " Le choix de "bleu" plutôt qu' "azur", m'avait semblé sauter davantage aux yeux et être plus quenaldien, tout en restant mystérieux dans sa signification par rapport au livre. [Queneau] m'a expliqué le sens français de l'expression, qui désigne de façon ironique les personnes romantiques, idéalistes, nostalgiques d'une pureté disparue, mais il ne m'a pas donné d'autres éclaircissements sur ce que recouvre cette image."

(12) Mario Fusco, Intervention à la Table ronde " Les Fleurs bleues de Raymond Queneau ", in Dix-septièmes assises de la Traduction littéraire, Arles, Atlas/Actes Sud, 2001, p. 40. 
des vertus anxiolytiques, hypnotiques et anticonvulsives. Leur utilisation chronique entraîne une dépendance physique et psychologique. C'est ce qui se passe notamment avec les habitués du Bar Biture. Le pastiche exige " une complicité avec le lecteur, il faut que le lecteur reconnaisse la source pour apprécier l'imitation qui n'est pas forcément parodique. Qui peut être un hommage. Qui peut être plus proche de l'imitation ou plus proche de la paraphrase. Le pastiche demande d'avoir l'oreille fine et juste, car chez un écrivain, quand on tient l'air, les paroles viennent vite(13) ". La compréhension du pastiche présuppose, certes, une certaine culture littéraire, mais la traduction de Bar Biture ne pose en soi aucun problème et Calvino propose à juste titre Bar Biturico.

L'extrait suivant résume la résolution de certaines difficultés traductionnelles mentionnées cidessus :

\begin{tabular}{|c|c|}
\hline Les $\mathrm{f}$ & I fio \\
\hline $\begin{array}{l}\text { Là-dessus, on apporta l'essence de fenouil et } \\
\text { l'eau plate. Ils burent. } \\
\text { - Et comment nomadez-vous? demanda Cidrolin. } \\
\text { À pied, à cheval, en voiture? en hélico, en vélo, } \\
\text { en auto? } \\
\text { - En stop, répondit la fille. } \\
\text { - En auto-stop? } \\
\text { - Bien sûr en auto-stop. } \\
\text { - Moi, je voyage parfois en autotaxi. C'est moins } \\
\text { économique. } \\
\text { - L'argent on s'en fout. } \\
\text { - D'accord. Et mon essence de fenouil ? } \\
\text { - Pas mauvais. Je préférons l'eau pure. }\end{array}$ & $\begin{array}{l}\text { In quella, venne servita l'essenza di finocchio et } \\
\text { l'acqua naturale. Bevvero. } \\
\text { - E com'è che nomandate ? - domandò Cidro- } \\
\text { lin. - A piedi, a cavallo, in macchina? in bici, } \\
\text { moto, auto, elico? } \\
\text { - In stop, - rispose la ragazza. } \\
\text { - Autostop? } \\
\text { - Certo, icciaíking. } \\
\text { - lo l'autostop lo faccio solo coi tassi. Ė più caro. } \\
\text { - II denaro me ne frego. } \\
\text { - D'accordo. E la mia essenza di finocchio? } \\
\text { - Mica male. lo, meglio l'acqua pura. }\end{array}$ \\
\hline
\end{tabular}

Laissons de côté le fait que cette conversation ressemble à un dialogue de fous. Les mots en italique mettent en évidence les libertés prises par Calvino vis-à-vis de l'original, qui peuvent être réduites à quelques stratégies : mots rajoutés (" en hélico, en vélo, en auto " - in bici, moto, auto, elico - la séquence est complétée par " moto " afin de préserver l'assonance) ; substitution de certains éléments par leurs contraires ("C'est moins économique "- $\dot{E}$ più caro); modification de l'ordre des mots ("Moi, je voyage parfois en autotaxi " - lo l'autostop lo faccio solo coi tassi) ; changement de la personne grammaticale (" on s'en fout "-me ne frego); refus de traduire les constructions agrammaticales ("Je préférons l'eau pure " - lo, meglio l'aqua pura) ; création de néologismes pour transmettre le fait de style (" en auto-stop "-

(13) Marie-Claire Pasquier, "Ezra Pound traducteur ", Vingtièmes assises de la traduction littéraire, Arles, Atlas/Actes Sud, 2004, p. 28. 
icciaiking). Calvino se permet de " joyciser " de temps en temps les équivalents proposés pour augmenter l'effet comique. Ainsi, au mot italien il préfère le mot anglais, hitchhike avec le suffixe -ing, mais écrit d'une façon invraisemblable, à l'italienne. Ou bien, il opte pour le terme de campinghe (p. 8) au lieu de campeggio pour tenir compte de la déformation de la graphie " camping " dans l'original (" campigne ", p. 19)(14). Bref, ce ne sont que quelques preuves du fait que rien n'échappe à ce lecteur très attentif et à ce traducteur très respectueux de l'œuvre originale et de son auteur.

Je voudrais m'arrêter aussi sur la fameuse " essence de fenouil " derrière laquelle il y a toute une histoire. Évidemment, cette liqueur n'existe pas. Mais le fenouil est une plante de la famille de l'anis qui est la matière première de l'anisette. La boisson préférée de Cidrolin, le personnage dont le nom renvoie au cidre, est normalement coupée avec de l'eau plate. J'ouvre une parenthèse pour souligner la décision de Calvino de traduire l'" eau plate " par " acqua naturale " qui fait penser plutôt à l'eau minérale qu'à l'eau du robinet. En tout cas, l'" essence de fenouil " renvoie aux alcools qui contiennent des extraits de plantes de la même famille et ont une renommée plus ou moins douteuse, à savoir l'absinthe, l'anisette, le pastis, le Pernod. Queneau précise, en outre, de manière récurrente, que la marque d'essence de fenouil favorite de son personnage est le Cheval blanc, que Calvino traduit littéralement par Cavallo Bianco : l'allusion à la célèbre marque de whisky White Horse ne peut pas passer inaperçue.

D'ailleurs, la traduction de "l'essence " est loin d'être évidente, malgré les apparences, du fait de la polysémie : le mot italien essenza n'implique pas toutes les connotations de l'" essence ", notamment l'extrait d'une substance, le carburant, l'espèce d'arbre, la nature des choses. D'autre part, Queneau joue sur l'homophonie "l'essence " / "les sens ", ce qui ajoute un autre niveau signifiant à l'expression. Cette connotation se perd dans l'essenza di finocchio. Or, Calvino arrive à compenser partiellement cette perte par la connotation d'homosexuel que le mot italien finocchio présente. II réussit donc à entrer dans le jeu de Queneau pour sauver la polysémie et l'effet comique qui en découle, en faisant preuve d'un sens linguistique et d'une virtuosité traductionnelle sans pareils.

\section{Traduire l'effet ludique : le cas de l'expression corporelle}

La traduction des locutions, des tournures et des métaphores constitue, en général, une pierre d'achoppement. La traduction littérale étant rarement une solution convenable, on recourt systématiquement à l'équivalence fonctionnelle. Les choix de Calvino, concernant la réinvention de l'expression corporelle dans les Fleurs bleues, concernent essentiellement les jeux de mots, les métaphores personnelles, les configurations insolites. Pour mettre un peu d'ordre dans

(14) Cf. Sergio Cappello, Les Années parisiennes d'Italo Calvino (1964-1980) Sous le signe de Raymond Queneau, Paris, Presses de l'Université Paris-Sorbonne, 2007, p. 176. 
les partis pris repérés dans le texte-cible, j'opte pour leur répartition en quelques catégories, à savoir expressions neutres, expressions familières, expressions agrammaticales, expressions figurées non lexicalisées, expressions figées. Pour ce faire, je vais analyser deux passages où les problèmes de la traduction des éléments corporels sont magistralement résolus.

Le premier est un extrait du chapitre III :

\begin{tabular}{|c|c|}
\hline Les fleurs bleues, p. 40 & I fiori blu, p. 30 \\
\hline $\begin{array}{l}\text { Après avoir dit ses patenôtres et soulagé sa ves- } \\
\text { sie dans l'escalier, il [le duc d'Auge] se dirigea } \\
\text { vers la chapelle pour y entendre la messe dite } \\
\text { par son chapelain Onésiphore Biroton. Onési- } \\
\text { phore Biroton était un abbé de choc ; si le duc lui } \\
\text { flanquait un coup de pied, il en rendait deux, } \\
\text { aussi le duc l'aimait-il fort et, ce jour-là, il avait } \\
\text { hâte de s'entretenir avec lui de plusieurs points } \\
\text { importants. L'abbé Biroton n'eut pas plutôt ita- } \\
\text { missaesté que le duc d'Auge l'entraîna sur le } \\
\text { baile et lui dit: } \\
\text { - Écoute-moi bien, Onésiphore, j'ai moult soucis } \\
\text { que je te vais déverser dans les oreilles. }\end{array}$ & $\begin{array}{l}\text { Detti i suoi paternostri, allegerita la vescica per } \\
\text { le scale, si diresse in cappella a sentir messa. II } \\
\text { cappellano Onesiforo Biroton era un prete del } \\
\text { clero d'assalto; se il Duca gli mollava un calcio } \\
\text { lui gliene rendeva due, e perciò il Duca l'aveva in } \\
\text { gran simpatia. Quel giorno, il Duca non vedeva } \\
\text { l'ora d'intrattenersi con l'abate su vari punti } \\
\text { importanti. Non appena Don Biroton itamis- } \\
\text { saestò, il Duca d'Auge se lo tirò dietro sugli spalti } \\
\text { e gli disse: } \\
\text { - Ascoltami bene, Onesiforo, dimolti pensieri il } \\
\text { mio animo alberga che ora nelle orecchie vado } \\
\text { a mescerti. }\end{array}$ \\
\hline
\end{tabular}

Les trois traductèmes retenus en l'occurrence sont : " soulagé sa vessie ", "flanquait un coup de pied ", " déverser dans les oreilles ". N'oublions pas qu'il s'agit d'un texte oulipien et que par conséquent les " oulipèmes [...] doivent être traités et traduits comme des unités de traduction insécables, non divisibles sémantiquement bien que divisibles syntaxiquement "(15). Et que la traduction « réinventive » de Calvino vise la fidélité au texte de Queneau, entendue surtout comme fidélité à l'esprit (mais aussi autant que possible à la lettre) de l'original. Les équivalents proposés par le traducteur-écrivain produisent leur effet non pas tellement grâce à la reprise ponctuelle de la "chose " traduite, mais grâce aux évocations qu'ils suscitent : les mêmes que sous-entendait Queneau. Ainsi, Calvino traduit " après avoir [...] soulagé sa vessie " par allegerita la vescica, en recourant à quelque transformation morphosyntaxique ; " flanquait un coup de pied " par mollava un calcio (le mot calcio implique le pied), en préservant le registre stylistique ; " je te vais déverser dans les oreilles " par ora nelle orecchie vado a mescerti, en atténuant légèrement le registre familier de l'expression et en rétablissant la correction grammaticale.

(15) Georgiana Lungu Badea, "Traductions transfuges, oulipotentielles ", op. cit., p. 77. 
Et voilà maintenant le début du chapitre IV :

\begin{tabular}{|c|c|}
\hline Les fleurs bleues, p. 48 & I fic \\
\hline $\begin{array}{l}\text { À la terrasse du café, des couples pratiquaient le } \\
\text { bouche à bouche et la salive dégoulinait le long } \\
\text { de leurs mentons amoureux ; parmi les plus } \\
\text { acharnés à faire la ventouse se trouvaient } \\
\text { Lamélie et un ératépiste, Lamélie surtout, car } \\
\text { l'ératépiste n'oubliait pas de regarder sa montre } \\
\text { de temps à autre vu ses occupations profes- } \\
\text { sionnelles. Lamélie fermait les yeux et se consa- } \\
\text { crait religieusement à la linguistique. } \\
\text { Vint la minute de séparation ; l'ératépiste } \\
\text { commença lentement les travaux de décollement } \\
\text { et, lorsqu'il fut parvenu à ses fins, cela fit flop. II } \\
\text { s'essuya du revers de la main et dit: } \\
\text { - Faut que je me tire. } \\
\text { Et il répandit un peu de bière sur ses muqueuses } \\
\text { asséchées. }\end{array}$ & $\begin{array}{l}\text { Nel caffè all'aperto la saliva traboccava giú per } \\
\text { gli amorosi menti delle coppiette intente al bocca } \\
\text { a bocca; tra i più accaniti a far ventosa si trova- } \\
\text { vano a essere Lamelia e un dipendente trasporti } \\
\text { publici, soprattutto lei, perché il dipendente } \\
\text { trasporti publici non dimenticava di guardare di } \\
\text { tanto in tanto l'orologio in relazione alle proprie } \\
\text { mansioni professionali. A occhi chiusi, Lamelia si } \\
\text { consacrava religiosamente alla linguistica. } \\
\text { Venne il momento del distacco; il dipendente } \\
\text { trasporti publici diede lentamente inizio alle ope- } \\
\text { razioni di decollo dall'incollamento orale, e una } \\
\text { volta portatele a buon fine con un: flopp! s'asciugò } \\
\text { sul rovescio della manica, disse: - Bisogna che } \\
\text { scappi, - e s'asperse di birra le mucose } \\
\text { inaridite. }\end{array}$ \\
\hline
\end{tabular}

En ce qui concerne la deuxième série d'expressions corporelles, reprenons le classement défini tout à l'heure, en y insérant les trois unités relevées dans l'extrait précédent :

- expressions neutres : " ses muqueuses asséchées " - le mucose inaridite ; "mentons amoureux " - amorosi menti (le mot menti rajoute une dimension polysémique car il signifie " mentons " et " esprits ") ; " II s'essuya du revers de la main " - s'asciugò sul rovescio della manica (déplacement métonymique de la " main " à la " manche ") ;

- expressions agrammaticales : "je te vais déverser dans les oreilles " - ora nelle orecchie vado a mescerti ;

- expressions familières : "flanquait un coup de pied "-mollava un calcio ; " déverser dans les oreilles " - nelle orecchie vado a mescerti ;

- expressions figurées non lexicalisées : "les travaux de décollement [des bouches] " - operazioni di decollo dall'incollamento orale (traduction explicite et explicative) ; " Lamélie fermait les yeux et se consacrait religieusement à la linguistique [entendue littéralement comme travail de la langue] "-A occhi chiusi, Lamelia si consacrava religiosamente alla linguistica ; " après avoir [...] soulagé sa vessie " - allegerita la vessica,

- expressions figées : "le bouche à bouche " - il bocca a bocca ; " faire la ventouse " - far ventosa. 
Évidemment, dans les extraits cités, comme d'ailleurs dans toute sa traduction, Calvino recourt forcément à la modification de l'ordre des mots, à l'italianisation des noms des personnages (Lamélie - Lamelia, Onésiphore - Onesiforo), à la " joycisation " (" itamissaesté " - itamissaestò). Ajouts, substitutions ou omissions ne manquent pas, mais ces déformations sont toujours justifiées et conformes à son projet.

La qualité de sa traduction réside, entre autres, dans la réinvention dans la langue-cible du style quenaldien, en préservant la légèreté de l'humour caractéristique de Queneau. Les équivalents qu'il propose, puisés dans l'italien ou fruits de son propre génie de traducteur-écrivain, parviennent à rendre superbement dans le texte-cible les effets voulus. Mario Fusco fait à ce sujet une remarque très juste: "En général il traduit le sens de la citation ; éventuellement il garde le rythme ; il ne donne jamais d'indications(16). "

Pour conclure, je voudrais souligner que cette version des Fleurs Bleues potentialise l'œuvre originale. Elle se présente décidément comme une traduction "rajeunissante " et non pas comme une "traduction-photographie ", pour reprendre les termes d'Ezra Pound(17) ou bien comme une traduction " domestiquée " et non pas "étrangéisée ", pour reprendre ceux de Lawrence Venuti(18). Elle permet d'explorer de fond en comble le texte oulipien pour dépister ses tensions et d'entrer en harmonie avec son auteur pour partager ses intentions. Or, au dit de Walter Benjamin, seule une interprétation attentive et sensible est capable d'assurer la survie et le renouvellement de l'original. La version des Fleurs Bleues qu'offre Italo Calvino est incontestablement une interprétation soignée. Ses Fiori blu demeurent toujours un bel exemple de traduction " réinventive " extrêmement amusante, attrayante et captivante.

irena_kristeva@yahoo.com

(16) Mario Fusco, Intervention à la Table ronde "Les Fleurs bleues de Raymond Queneau ", op. cit., p. 38.

(17) Cf. Ezra Pound, "Guido's Relations ", in Lawrence Venuti (dir.), The Translation Studies Reader, London and New York, Routledge, 2000, p. 33.

(18) Cf. Lawrence Venuti, The Translator's Invisibility. A History of Translation, London \& New York, Routledge, 1995, p. 34. 


\section{Bibliographie}

BOLLACK Jean, 2000. Sens contre sens. Comment lit-on ? Paris, éditions La passe du vent.

CAPPELLO Sergio, 2007. Les Années parisiennes d'Italo Calvino (1964-1980). Sous le signe de Raymond Queneau. Paris, Presses de l'Université Paris-Sorbonne.

FREUD Sigmund, 1971. Le Mot d'esprit et ses rapports avec l'inconscient, trad. fr., Paris, Gallimard.

FUSCO Mario, Intervention à la table ronde "Les Fleurs bleues de Raymond Queneau ", in Dixseptièmes assises de la traduction littéraire. Arles, Atlas/Actes Sud, 2001, p. 31-48.

GENETTE Gérard, 1994. L'OEuvre de l'art I, Immanence et transcendance. Paris, Le Seuil.

LUNGU BADEA Georgiana, "Traductions transfuges, oulipotentielles ", in Translationes, $n^{\circ}$, 2013, p. 75-81.

PASQUIER Marie-Claude, "Ezra Pound traducteur ", Vingtièmes assises de la traduction littéraire. Arles, Atlas/Actes Sud, 2004, p. 13-34.

POUND Ezra, "Guido's Relations ", in Lawrence Venuti (ed.), The Translation Studies Reader. London and New York, Routledge, 2000, p. 26-33.

QUENEAU Raymond, 1965. Les Fleurs bleues. Paris, Gallimard, coll. " Folio ".

QUENEAU Raymond, 1995. I fiori blu. tr. it. di Italo Calvino, Torino, Einaudi tascabili.

STEINER George, 1978. Après Babel. Une poétique du dire et de la traduction, trad. fr., Albin Michel.

VENUTI Lawrence, 1995. The Translator's Invisibility. A History of Translation. London \& New York, Routledge.

Irène Kristeva est professeure associée à la Faculté des Lettres classiques et modernes de l'Université de Sofia. Elle y enseigne la Théorie de la traduction et la Littérature française contemporaine. Titulaire d'un doctorat de Sémiologie du Texte et de l'Image, délivré par I'Université de Paris 7, elle est notamment l'auteur de Pascal Quignard : la fascination du fragmentaire (L'Harmattan, 2008), Pour comprendre la traduction (L'Harmattan, 2009) et Les Métamorphoses d'Hermès (en bulgare, Est-Ouest, 2015). Traductrice du français et de l'italien, elle a traduit et supervisé la traduction, entre autres, des œuvres de Leopardi, Pascal, Quignard, Bourdieu, Bobbio, Deleuze, Derrida, Eco, Foucault, Ricœur. Auditrice libre de l'association Espace Analytique.

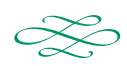

